\title{
La naturaleza dual del proceso diagnóstico y su vulnerabilidad a los sesgos cognitivos
}

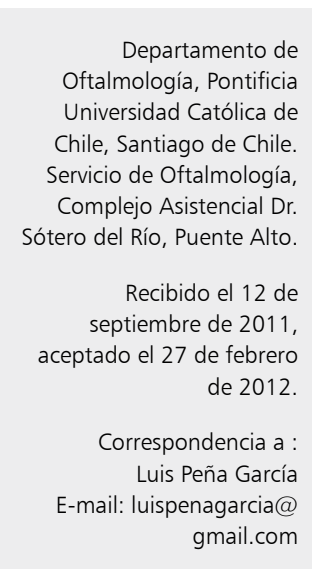

LUIS PEÑA G.

\section{Description of clinical thinking by the dual-process theory}

Clinical thinking is a very complex process that can be described by the dualprocess theory, it has an intuitive part (that recognizes patterns) and an analytical part (that tests hypotheses). It is vulnerable to cognitive bias that professionals must be aware of, to minimize diagnostic errors.

(Rev Med Chile 2012; 140: 806-810).

Key words: Diagnosis, dual (Psychiatry); Diagnostic errors; Diagnostic techniques and procedures.
"La vida es breve; el arte, extenso; la oportunidad, fugaz; la experiencia, insegura; el juicio, difícil."

Hipócrates, aforismo $\mathrm{n}^{\circ} 1^{1}$.

A veces llegamos al diagnóstico correcto en forma muy rápida y casi sin esfuerzo. En otras ocasiones no encontramos una solución en forma inmediata y debemos detenernos a pensar en el problema clínico, volver a interrogar al paciente, examinarlo otra vez con más detención, sopesar las probables causas de su problema, solicitar algunos exámenes complementarios y luego de un análisis consciente y detenido, a veces llegamos a un diagnóstico. Desgraciadamente los médicos podemos cometer errores diagnósticos y ningún médico está libre de ellos ${ }^{2,3}$.

Los problemas clínicos que enfrentamos pueden ser muy complejos, con numerosas variables en juego, además se nos presentan mal estructurados: la información clínica inicial puede ser incompleta, conflictiva o ambigua. Para hacerlo más complicado, el problema clínico puede ir cambiando en el tiempo, al aparecer nuevos síntomas y signos.

Para hacer un diagnóstico, el médico cuenta con sus conocimientos y habilidades clínicas, pero estas no son suficientes, porque existen muchas formas de llegar al diagnóstico y muchas de pasarlo por alto. Se necesita un método para aplicar el conocimiento y orientar la anamnesis y el examen físico. El médico debe pensar.

Revisaremos cómo el pensamiento médico durante el diagnóstico puede ser descrito por la teoría dual de la cognición, la estrecha vinculación entre conocimiento experiencial y diagnóstico, y la importancia de los sesgos cognitivos en el error diagnóstico.

\section{Modelo de razonamiento clínico de Barrows ${ }^{4}$}

En una fase precoz del contacto entre el médico y el paciente, el médico se forma un concepto inicial del paciente, basado en su motivo de consulta, sus características demográficas y la información que entrega en forma espontánea. En base a este concepto inicial, el médico se formula una serie de hipótesis iniciales, que guían su investigación clínica (anamnesis y examen físico). En forma paralela a la anamnesis y examen físico se va haciendo un análisis de la información obtenida en tiempo real, que va descartando hipótesis, consolidando otras o generando hipótesis nuevas.

Cuando se completa la investigación clínica y análisis, el médico ya tiene en su mente una síntesis del problema,que contiene los hallazgos relevantes descritos en términos médicos (que funcionan como cualificadores semánticos). De la síntesis 
del problema pasa a tomar una decisión diagnóstica, es decir, ha seleccionado la hipótesis más probable, a la que denominamos diagnóstico de trabajo, diagnóstico tentativo o impresión diagnóstica. Si existen otras hipótesis también dentro del marco de lo posible, pero que con la información disponible nos parecen menos probables, las agrupamos en una lista que denominamos diagnóstico diferencial. Si la información recopilada es lo suficientemente potente para afirmar con certeza el diagnóstico, hablamos de diagnóstico final.

Aunque el esquema de razonamiento clínico se ajusta a lo que debería ser el trabajo médico, deja muchas incógnitas sobre la intimidad del proceso cognitivo durante el diagnóstico, las que pueden ser explicadas por la teoría del procesamiento dual.

\section{Teoría del procesamiento dual ${ }^{5-7}$}

De acuerdo a esta teoría se utilizan dos sistemas cognitivos en el razonamiento: el sistema intuitivo y el sistema analítico, que trabajan en forma complementaria (Figura 1).

El razonamiento se inicia con la activación automática del sistema intuitivo, que evalúa el contexto y la información inicial, trabaja por debajo del nivel de conciencia, reconociendo patrones y generando hipótesis en forma rápida ${ }^{8}$. La generación de hipótesis en forma intuitiva es tan importante que si la hipótesis correcta es generada en los primeros cinco minutos, hay $95 \%$ de posibilidades de llegar al diagnóstico correcto, en

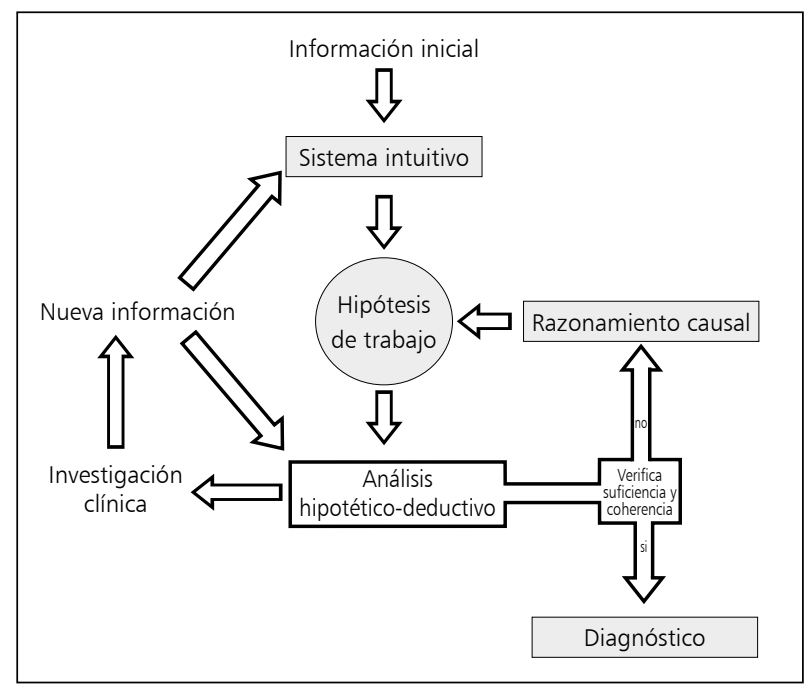

Figura 1. Esquema del diagnóstico médico como procesamiento dual. cambio si la hipótesis correcta no se genera en los primeros cinco minutos, hay $95 \%$ de posibilidades de no llegar al diagnóstico correcto $^{6}$.

En una segunda etapa el sistema analítico examina las hipótesis generadas por el sistema intuitivo, a través de un sistema consciente y estructurado, el análisis hipotético-deductivo, con el que se verifica si los atributos de la hipótesis están presentes en el problema clínico (coherencia) y si estos explican todos los componentes del problema clínico (suficiencia), así va descartando unas hipótesis y afirmando otras ${ }^{8}$.

Si en este punto nos quedamos sin hipótesis, podemos usar otra función del sistema analítico, llamada razonamiento causal, que genera nuevas hipótesis en forma consciente. La forma en que se representa el caso clínico es clave, especialmente el uso de los cualificadores semánticos en la síntesis del problema, que son usados como índices de memoria para buscar asociaciones relevantes con conocimientos de la anatomía y fisiopatología. El razonamiento causal es muy importante en el análisis de problemas con los que no se tiene experiencia, nuevos, complejos o atípicos ${ }^{8}$.

A pesar de que ambos sistemas son complementarios, existen situaciones en que el razonamiento es primordialmente intuitivo o analítico. Por ejemplo, en casos rutinarios el sistema intuitivo rápidamente llega a una conclusión con un altísimo nivel de certeza y se requiere de muy poco procesamiento analítico. En cambio, en casos donde el problema que se nos presenta es nuevo, complejo o mal definido, el trabajo del sistema analítico es indispensable y mucho más arduo.

En todo caso, el reconocimiento de pautas es claramente fundamental para el éxito del razonamiento médico, por lo que lo discutiremos en detalle.

\section{Reconocimiento de pautas}

Cuando generamos una hipótesis diagnóstica, lo que hacemos es identificar ciertos atributos del problema médico del paciente como una categoría específica (el diagnósti- 
co). Evidentemente para llegar a un diagnóstico, el médico debe tener un conocimiento previo de él. En este sentido, se distinguen dos tipos de conocimiento médico: el conocimiento conceptual y el experiencial ${ }^{9,10}$.

\section{Conocimiento de bajo nivel o conceptual}

Es el conocimiento de los contenidos, incluye todo lo que debe saber un médico: anatomía, fisiopatología, etiología, síntomas, signos, diagnósticos, síndromes, pronóstico, tratamiento. Cuando el novato atiende por primera vez a un paciente con un problema específico, debe hacer un esfuerzo consciente por relacionar los signos y signos que encuentra con su conocimiento de contenidos para llegar a un diagnóstico ${ }^{11,12}$. El conocimiento conceptual es fácil de olvidar.

\section{Conocimiento de alto nivel o experiencial}

La experiencia potencia la retención de los conocimientos en la memoria de largo plazo, y facilita la formación de una red de asociaciones. En esta red los contenidos no sólo se consolidan, sino que adquieren significado, relevancia y contexto ${ }^{12,13}$.

El conocimiento experiencial consiste en modelos mentales ${ }^{13}$ (guiones, pautas o scripts) en varios formatos, pueden ser de carácter general como el conocimiento sobre un síndrome o una enfermedad, pero también puede consistir en un conjunto de "casos clínicos" de pacientes específicos atendidos con ese diagnóstico. Estos formatos de representación tienen un efecto sinérgico y dado que los casos clínicos son almacenados como episodios autobiográficos, son fácilmente accesibles, incluso después de muchos años. Todo parece indicar que la capacidad de recordar pacientes vistos no es sólo una curiosidad de la mente del médico, sino probablemente una característica central de la cognición del experto y de su capacidad de reconocimiento de pautas.

Cuando atendemos a un paciente, los modelos mentales se empiezan a activar en forma automática, incluso en una etapa muy precoz del encuentro médico-paciente, en la medida que nuestro sistema intuitivo reconoce pautas que ya estaban almacenadas en nuestra memoria de largo plazo como conocimiento experiencial. Los modelos mentales nos permiten lograr una comprensión global del problema, pese a que su presentación sea incompleta o fragmentaria ${ }^{13}$.

Los modelos mentales activados en forma intuitiva y automática son las hipótesis iniciales, que posteriormente son procesadas en forma consciente por el sistema analítico.

La forma en que los médicos presentan los casos a sus colegas está muy influida por los modelos mentales que poseen. Mientras que los alumnos y los médicos novatos recitan enormes cantidades de datos sobre el paciente, los médicos experimentados resumen el problema del paciente utilizando la información clínica relevante.

El conocimiento biomédico de bajo nivel sigue siendo utilizado. Por una parte provee un criterio de coherencia para las hipótesis. Pero más importante, cuando no se encuentran modelos mentales atingentes a un caso clínico complejo, el conocimiento biomédico puede ser usado para entender la situación y para generar hipótesis a través de una cadena de razonamiento causal ${ }^{8,11}$.

Los modelos mentales de las enfermedades son estructuras dinámicas, que van cambiando, especialmente durante los primeros años de práctica médica. Cuando un médico o estudiante atiende a un paciente con una enfermedad que no había visto antes, aunque la haya estudiado, requerirá bastante esfuerzo cognitivo para llegar a un diagnóstico. Pero la segunda vez que se enfrente a la misma enfermedad su eficiencia será mayor, pues ya tendrá un modelo mental de ella gracias a su experiencia previa. En esta etapa la manera más efectiva de construir estos modelos mentales es a través de la experiencia clínica monitorizada ${ }^{14}$. En la medida que el médico atiende cada vez más pacientes va construyendo modelos más eficientes, hasta llegar a modelos muy depurados y ricos en asociaciones $^{9-14}$.

\section{Fallas cognitivas}

El razonamiento, especialmente el intuitivo, es afectado por las reacciones emocionales negativas del médico hacia el paciente, como a veces ocurre con un drogadicto o un delincuente, frente a pacientes manifiestamente disgustados o agresivos y desgraciadamente, como muchos estudios indican, a veces también pasa con pacientes con enfermedades psiquiátricas, e incluso frente a obesos mórbi$\operatorname{dos}^{3}$. El razonamiento médico también puede ser obnubilado por los sentimientos eróticos. Otros factores perjudiciales son exceso de confianza, baja autoestima, desilusión, complacencia y falta de motivación. 
El razonamiento, especialmente el analítico, también depende mucho del nivel de alerta del médico ${ }^{6}$ y puede ser afectado por factores como la fatiga, falta de sueño, distracción, otras preocupaciones y apuro.

\section{Sesgos cognitivos}

Reflejan una inclinación a pensar de cierta manera, habitualmente involuntaria e inconsciente ${ }^{15}$. Para minimizar sus consecuencias negativas debemos tener una disposición analítica, disciplinada y consciente para monitorizar nuestra forma de pensar ${ }^{16}$. A continuación se enuncian y describen algunos de los sesgos y errores cognitivos más frecuentes ${ }^{17-20}$.

El cierre prematuro uno de los errores cognitivos más frecuentes. Consiste en la tendencia a terminar el proceso de razonamiento y toma de decisiones en forma prematura, aceptando un diagnóstico que no ha sido verificado por completo. Sigue la máxima "cuando se hace el diagnóstico, el pensamiento termina".

La satisfacción de búsqueda es la tendencia a dejar de buscar cuando ya se ha encontrado algo. Por ejemplo, se pasa por alto una segunda fractura en una radiografía, pues la búsqueda se da por satisfecha al encontrar la primera fractura.

El sesgo de confirmación consiste en la tendencia a buscar y darle más peso a la evidencia que confirma nuestro diagnóstico, y no buscar o darle menos peso a la evidencia que lo refuta.

El exceso de confianza es la tendencia a creer que sabemos más de lo que realmente sabemos. Cuando hay exceso de confianza se tiende a actuar con información incompleta, se coloca mucha fe en las impresiones iniciales sin examinar cuidadosamente la evidencia.

El anclaje es la tendencia a aferrarse a características relevantes que aparecen en forma precoz en el proceso diagnóstico y formarnos una impresión inicial del paciente basados en ellas, sin ajustar esta impresión diagnóstica a la luz de la información que va apareciendo después. Está en estrecha relación con el efecto de orden, es decir, tendemos a recordar más el comienzo (efecto de primacía) y el final (más reciente).

El sesgo de disponibilidad es la tendencia a creer que algo es más frecuente o más probable, porque se nos viene fácilmente a la mente. La experiencia reciente con una enfermedad aumenta la probabilidad de que esta sea diagnosticada, también es menos probable diagnosticar una enfermedad que hace mucho tiempo no hemos visto.

La ley de Sutton consiste en que tendemos a creer en lo obvio, sin considerar otras posibilidades con la suficiente acuciosidad.

El momentum diagnóstico es la tendencia a creer en los diagnósticos ya hechos por otra persona. Una vez que la etiqueta de un diagnóstico se ha colocado en un paciente, esta se hace cada vez más pegajosa y es difícil sacarla de nuestra mente.

La apuesta de frecuencia es la tendencia a optar por un diagnóstico sobre la base de que es más frecuente. La apuesta de frecuencia se puede resumir en el aforismo "cuando escuches ruido de cascos, piensa en caballos, no en cebras".

Las tareas rutinarias y repetitivas con frecuencia hacen que nuestro pensamiento siga estilos ortodoxos, en vez de favorecer el pensamiento lateral. A esto se le denomina pensamiento en línea vertical. Una estrategia efectiva para evitar este sesgo es pensar "que otro diagnóstico podría ser", lo que estimula la creatividad y el pensamiento lateral, o plantearse "qué es lo peor que podría ser" para no pasar por alto un diagnóstico raro pero serio (estrategia del peor escenario).

Error de representatividad. Consiste en que se buscan las manifestaciones del prototipo de la enfermedad. Este tipo de error hace que las variantes atípicas de una enfermedad no sean diagnosticadas.

Sesgo de multiplicidad. Cuando pensamos en muchas hipótesis al mismo tiempo nos confundimos. Nuestro pensamiento funciona mejor considerando hasta cuatro hipótesis a la vez.

Sesgo de pronóstico. Tendencia a optar por diagnósticos que tienen buen pronóstico. Ocurre especialmente cuando el médico tiene un lazo afectivo con el paciente, y refleja su tendencia a creer más en lo que él desea creer (que la enfermedad sea benigna).

Retirada de la cebra. Ocurre cuando un diagnóstico raro (la cebra, en vez del caballo), figura prominentemente en el diagnóstico diferencial, pero por alguna razón el diagnóstico se retrasa o no se hace. A veces el médico carece de la confianza suficiente para mantener el diagnóstico, $o$ está cansado o distraído, o puede tener recelos de emprender exámenes o procedimientos caros o invasivos, o no quiere contradecir a otros 
miembros del equipo que piensan diferente, o no quiere parecer como el que propone diagnósticos esotéricos, etc.

\section{Conclusión}

El razonamiento médico durante el diagnóstico puede ser descrito como un proceso dual, tiene un componente intuitivo que genera hipótesis utilizando asociaciones de memoria automáticas y depende fuertemente del conocimiento experiencial, y un componente analítico, que procesa esas hipótesis validándolas o descartándolas y cuando es necesario genera nuevas hipótesis utilizando el razonamiento causal. En los casos rutinarios, el sistema intuitivo encuentra el diagnóstico en forma rápida, pero sus conclusiones deben ser siempre revisadas por el sistema analítico. Ambos sistemas funcionan de manera complementaria y nos permiten hacer diagnósticos correctos la mayoría de las veces, a pesar de la complejidad de los problemas clínicos.

Pero incluso los mejores médicos cometen errores. Muchos son evitables. Para minimizar los errores diagnósticos debemos pensar acerca de cómo pensamos, monitorizar nuestro razonamiento y estar atentos a los errores cognitivos que podemos cometer en forma inconsciente.

Porque en medicina la oportunidad es fugaz y la experiencia es insegura, debemos esforzarnos para que nuestro juicio sea claro.

\section{Referencias}

1. Hipócrates. Tratados, Editorial Gredos, 2007.

2. Graber M, Gordon R, Franklin N. Reducing diagnostic errors in medicine: what's the goal? Acad Med 2002; 77: 981-92.

3. Groopman J. ¿Me está escuchando, doctor? Un viaje por la mente de los médicos. Ediciones RBA, 2008.

4. Barrows H, Pickell GC. Developing clinical problemsolving skills. Norton, 1991.
5. Norman G. Dual processing and diagnostic errors. Adv in Health SciEduc 2009; 14: 37-49.

6. Pelaccia T, Tardiff J, Triby E Charlin B. An analysis of clinical reasoning through a recent and comprehensive approach: the dual-process theory. Medical Education Online 2011; 16: 5890.

7. Schmidt H, Norman G, Boshuizen H. A cognitive perspective on medical expertise: theory and implications. Acad Med 1990; 65: 611-21.

8. Kassirer J, Wong J, Kopelman R. Manual de razonamiento clínico, Lippincott Willimas \& Wilkins, $2^{\text {da }}$ edición, 2011.

9. Bordage G. Elaborated knowledge: a key to successful diagnostic thinking. Acad Med 1994; 69: 883-5.

10. Bordage G. Why did I miss the diagnosis? Some cognitive explanations and educational implications. Acad Med 1999; 74: S138-43.

11. Bruin A, Schmidt H, Rikers R. The role of basic science knowledge and clinical knowledge in diagnostic reasoning: a structural equation modeling approach. Acad Med 2005; 80: 765-73.

12. Slotnick H. How doctors learn: education and learning across the medical-school-to-practice trajectory. Acad Med 2001; 76: 1013-26.

13. Charlin B, Tardiff J, Boshuizen H. Scripts and medical diagnostic knowledge: Theory and applications for clinical reasoning instruction and research. Acad Med 2000; 75: 182-190.

14. Kassirer J. Teaching clinical reasoning: case-based and coached. Acad Med 2010; 85: 1118-24.

15. Croskerry P. The importance of cognitive errors in diagnosis and strategies to minimize them. Acad Med 2003; 78: 775-80.

16. Croskerry P. The cognitive imperative: Thinking about how we think. Acad Emerg Med 2000; 7: 1223-31.

17. Croskerry P. Clinical cognition and diagnostic error: applications of a dual process model of reasoning. Adv in Health SciEduc 2009; 14: 27-35.

18. Elstein A. Heuristics and biases: selected errors in clinical reasoning. Acad Med 1999; 74: 791-4.

19. Croskerry P. Achieving quality in clinical decision making: Cognitive strategies and detection of bias. Acad Emerg Med 2002; 9: 1184-204.

20. Tversky A, Kahneman D. Judgment under Uncertainty: Heuristics and biases. Science 1974; 185: 1124-31. 